\title{
Ropivacaína em Bloqueio Peridural Torácico para Cirurgia Plástica *
}

\section{Thoracic Epidural Anesthesia with Ropivacaine for Plastic Surgery}

José Roberto Nociti, TSA ${ }^{1}$, Paulo Sérgio Mateus Serzedo, TSA ${ }^{2}$, Eduardo Barbin Zuccolotto, TSA ${ }^{2}$, Raul Gonzalez ${ }^{3}$

\begin{abstract}
RESUMO
Nociti JR, Serzedo PSM, Zuccolotto EB, Gonzalez R - Ropivacaína em Bloqueio Peridural Torácico para Cirurgia Plástica

Justificativa e Objetivos - O bloqueio peridural torácico constitui técnica de anestesia hipotensiva, capaz de reduzir o sangramento no campo operatório. O presente estudo não-comparativo tem por finalidade observar os resultados do bloqueio peridural torácico com ropivacaína a 0,5\% associado a sedação com infusão contínua de propofol em cirurgia plástica.
\end{abstract}

Método - Participaram do estudo sessenta pacientes do sexo feminino com idades entre 18 e 62 anos, estado físico ASA I ou II, submetidas a cirurgias plásticas combinadas envolvendo mama, abdômen, glúteos, lipoaspiração. Após punção peridural em $T_{9}-T_{10}$ ou $T_{10}-T_{11}$, receberam $40 \mathrm{ml}$ de solução de ropivacaína a $0,5 \%$ e sufentanil $15 \mu \mathrm{g}$. Doses subseqüentes do anestesia local foram administradas através de cateter quando necessárias. Sedação foi obtida com infusão venosa contínua de propofol 40 a $50 \mu \mathrm{g} \cdot \mathrm{kg}^{-1} \cdot \mathrm{min}^{-1}$. Foram avaliadas as características de instalação e regressão do bloqueio, a evolução dos parâmetros hemodinâmicos e respiratórios, a incidência de eventos adversos.

Resultados - O nível superior de bloqueio sensorial foi $T_{2}$ em 52 pacientes (86,6\%), $C_{4}$ em $4(6,6 \%)$ e $T_{3}$ em 4 (6,6\%). A média para o tempo de latência foi $9,1 \pm 8,2$ minutos. Obteve-se bloqueio motor grau $2 \mathrm{em} 61,7 \%$ das pacientes e grau $1 \mathrm{em}$ $38,3 \%$. A média para o tempo de regressão completa do bloqueio motor foi $377,9 \pm 68,5$ minutos. A média para o tempo da primeira queixa espontânea de dor foi 965,1 $\pm 371,3$ minutos. Os valores médios de PAS, PAD, PAM e FC decresceram significativamente em relação ao controle a partir dos 15 min após a injeção do anestésico local, caracterizando anestesia hipotensiva. Treze pacientes $(21,7 \%)$ que apresentaram PAS < $65 \mathrm{mmHg}$ e/ou PAM $<50 \mathrm{mmHg}$ receberam vasopressor (etilfedrina) e $4(6,7 \%)$ que apresentaram $F C<50$ $\mathrm{bpm}$ receberam atropina. Não foi necessária transfusão sangüínea em nenhum paciente.

Conclusões - O bloqueio peridural torácico com ropivacaína a $0,5 \%$ e sufentanil $15 \mu \mathrm{g}$, associado à sedação com propofol em

\footnotetext{
* Recebido do (Received from) CET/SBA da Santa Casa de Misericórdia de Ribeirão Preto, SP

1. Responsável pelo CET/SBA

2. Co-responsável pelo CET/SBA

3. Cirurgião Plástico, Chefe da "Clínica Raul Gonzalez", Hospital Afiliado do $C E T / S B A$
}

Apresentado (Submitted) em 17 de julho de 2001

Aceito (Accepted) para publicação em 09 de outubro de 2001

Correspondência para (Mail to):

Dr. José Roberto Nociti

Rua Stélio Machado Loureiro, 21 - Alto da Boa Vista

14025-470 Ribeirão Preto, SP

E-mail:carp@keynet.com,br

(C) Sociedade Brasileira de Anestesiologia, 2002 infusão contínua, constitui método de anestesia hipotensiva de boa qualidade para cirurgias plásticas combinadas envolvendo mama, abdômen, glúteos e lipoaspiração. A monitorização contínua dos parâmetros hemodinâmicos e respiratórios e o controle dos efeitos do bloqueio sobre estes parâmetros são indispensáveis para o sucesso e a segurança da técnica.

UNITERMOS: ANESTÉSICOS, Local: ropivacaína; CIRURGIA, Plástica; TÉCNICAS ANESTÉSICAS, Regional: peridural torácica

\section{SUMMARY}

Nociti JR, Serzedo PSM, Zuccolotto EB, Gonzalez R - Thoracic Epidural Anesthesia with Ropivacaine for Plastic Surgery

Background and Objectives - Thoracic epidural blockade is a method of hypotensive anesthesia able to reduce bleeding during surgery. This non-comparative study aimed at evaluating the results of thoracic epidural blockade with $0.5 \%$ ropivacaine associated to propofol continuous infusion sedation in plastic surgery.

Methods - Participated in this study 60 female patients aged 18 to 62 years, physical status ASA I or II, scheduled for combined plastic surgeries of breast, abdomen, gluteus and liposuction. After epidural puncture in $T_{9}-T_{10}$ or $T_{10}-T_{11}$, patients received 40 $\mathrm{ml}$ of $0.5 \%$ ropivacaine and $15 \mu \mathrm{g}$ sufentanil. Additional local anesthetic doses were administered through an epidural catheter, if necessary. Sedation was induced with 40 to 50 $\mu \mathrm{g} \cdot \mathrm{kg}^{-1} \cdot \mathrm{min}^{-1}$ propofol continuous infusion. Blockade installation and regression, hemodynamic and respiratory parameters and the incidence of adverse effects were investigated.

Results - Upper sensory block level was $T_{2}$ in 52 patients (86.6\%), $C_{4}$ in 4 (6.6\%), and $T_{3}$ in 4 (6.6\%). Mean onset time was $9.1 \pm 8.2 \mathrm{~min}$. Motor block grade 2 was obtained in $61.7 \%$ of patients, and grade 1 in $38.3 \%$. Mean time for complete motor block regression was $377.9 \pm 68.5 \mathrm{~min}$. Mean time for first spontaneous pain complaint was $965.1 \pm 371.3 \mathrm{~min}$. SBP, DBP, MBP and $H R$ mean values have significantly decreased as compared to control as from $15 \mathrm{~min}$ after local anesthetic injection, thus characterizing hypotensive anesthesia. Thirteen patients $(21.7 \%)$ with $S B P<65 \mathrm{mmHg}$ and/or $M B P<50 \mathrm{mmHg}$ received a vasopressor (ethylphedrine) and 4 patients $(6.7 \%)$ with $H R<$ $50 \mathrm{bpm}$ received atropine. No patient needed blood transfusion.

Conclusions - Thoracic epidural blockade with $0.5 \%$ ropivacaine and $15 \mu \mathrm{g}$ sufentanil associated to propofol continuous infusion sedation is a satisfactory hypotensive anesthesia technique for combined plastic surgeries involving breast, abdomen, gluteus and liposuction. Continuous monitoring of hemodynamic and respiratory parameters as well as controlling blockade effects on those parameters are critical for the success and safety of the technique.

KEY WORDS: ANESTHETICS, Local: ropivacaíne; ANESTHETIC TECHNIQUES, Regional: thoracic epidural; SURGERY, Plastic 


\section{INTRODUÇÃO}

A cirurgia plástica é um dos ramos da cirurgia que mais se beneficiam da hipotensão arterial induzida. São antigas as observações de que a manutenção da pressão arterial sistólica em até $65 \mathrm{mmHg}$ e da pressão arterial média em até $50 \mathrm{mmHg}$ com este procedimento é segura e reduz significativamente o sangramento no campo operatório ${ }^{1,2}$. Posteriormente evoluiu-se para o conceito de anestesia hipotensiva ${ }^{3}$ compreendendo métodos de anestesia que buscam redução do sangramento com segurança. Um destes métodos é o bloqueio peridural, graças à redução da pré-carga e da resistência vascular sistêmica que ele provoca e, no caso do bloqueio peridural torácico, também à diminuição da contratilidade miocárdica secundária ao bloqueio das fibras simpáticas para o coração ${ }^{4,5}$.

Por outro lado, têm sido obtidos bons resultados com a ropivacaína, novo anestésico local $(A L)$ do grupo das pipecolxilididas, em anestesia peridural com finalidade cirúrgica ${ }^{6}$, destacando-se a menor intensidade do bloqueio motor ${ }^{7}$ e o menor potencial para toxicidade sobre os sistemas cardiovascular e nervoso, em relação à bupivacaína ${ }^{8}$.

O objetivo deste estudo não-comparativo foi observar os resultados do bloqueio peridural torácico com ropivacaína em solução a $0,5 \%$ associado a sufentanil e sedação com infusão venosa contínua de propofol, em cirurgia plástica.

\section{MÉTODO}

O estudo obteve a aprovação da Comissão de Ética do Hospital e dele participaram 60 pacientes que obedeceram aos seguintes critérios de seleção:

1. Sexo feminino, idade igual ou superior a 18 anos;

2. Estado físico ASA I ou II;

3. Programadas para cirurgias plásticas eletivas combinadas, envolvendo mama, abdômen, glúteos e lipoaspiração.

A medicação pré-anestésica constou de flunitrazepam (30 $\mathrm{mg}$ ) por via oral 60 minutos antes do início previsto para a cirurgia.

Após cateterismo de veia de grosso calibre em membro superior e início de infusão de solução glicosada a 5\%, foi realizada punção do espaço peridural em $T_{9}-T_{10}$ ou $T_{10}-T_{11} \operatorname{com~a}$ paciente na posição sentada. O espaço peridural foi identificado através do teste da perda de resistência à injeção de ar. Foi administrado o volume de $40 \mathrm{ml}$ de solução de ropivacaína a $0,5 \%$ (correspondendo à dose de $200 \mathrm{mg}$ do $\mathrm{AL}$ ) adicionada de sufentanil $15 \mu \mathrm{g}$, à velocidade de injeção de 5 $\mathrm{ml} . \mathrm{min}^{-1}$. Em seguida foi introduzido cateter no espaço peridural 8 a $10 \mathrm{~cm}$ em sentido cefálico, através do qual era administrada dose adicional de ropivacaína $(15 \mathrm{ml}$ da solução a $0,5 \%$, correspondendo a $75 \mathrm{mg}$ ) sempre que a duração do procedimento o exigisse.
Após instalação do bloqueio peridural, procedeu-se à sedação com midazolam $\left(0,10 \mathrm{mg} \cdot \mathrm{kg}^{-1}\right)$, por via venosa, seguido de propofol em infusão venosa contínua à velocidade de $40 \mathrm{a}$ $50 \mu \mathrm{g} \cdot \mathrm{kg}^{-1} \cdot \mathrm{min}^{-1}$ durante todo o procedimento. Administrou-se rotineiramente oxigênio em fluxo de $1 \mathrm{a} 2 \mathrm{~L}$. $\mathrm{min}^{-1}$ através de cateter introduzido em cânula orofaringea (Guedel).

Foi realizada a monitorização por método não-invasivo de: pressão arterial sistólica (PAS), pressão arterial diastólica (PAD), pressão arterial média (PAM), freqüência cardíaca (FC), eletrocardiograma (ECG), freqüência respiratória (FR), saturação da hemoglobina pelo oxigênio no oxímetro de pulso $\left(\mathrm{SpO}_{2}\right)$. Foram registrados em protocolo os valores obtidos para os parâmetros hemodinâmicos nos seguintes momentos:

- pré-bloqueio peridural (controle);

- 5 minutos após o término da injeção do AL;

- 15 minutos após o término da injeção;

- 30 minutos após o término da injeção;

- a cada 30 minutos daí por diante.

A administração de fluidos per-operatórios foi feita de acordo com regra proposta por Jenkins e Giesecke ${ }^{9}$, para cirurgias superficiais maiores: 8 a $10 \mathrm{ml} \cdot \mathrm{kg}^{-1} \cdot \mathrm{h}^{-1}$, iniciando-se com solução de glicose a $5 \%$ até o volume de $500 \mathrm{ml}$ e continuando-se com solução salina balanceada (Ringer com lactato) e solução de glicose a $5 \%$, nunca ultrapassando a dose total de $125 \mathrm{~g}$ de glicose. Nos casos de lipoaspiração úmida, foi praticada pelo cirurgião infiltração das áreas a serem aspiradas com solução fisiológica contendo lidocaína a $0,1 \%$ e adrenalina a $1: 1.000 .000^{10}$. Como uma fração superior a $80 \%$ do volume líquido infiltrado acaba sendo absorvida e entra na circulação ${ }^{11,12}$, esta quantidade foi descontada do volume calculado pela regra de Jenkins e Giesecke, administrando-se portanto um volume final de fluidos menor que o previsto.

Aavaliação do bloqueio sensorial foi feita da seguinte maneira:

a) Tempo de Latência - foi considerado o tempo decorrido entre o final da injeção e a ausência de dor à estimulação com pinça cirúrgica na área sensitiva correspondente ao nível de punção;

b) Nível Superior de Bloqueio - por estimulação cutânea com pinça cirúrgica com dente em intervalos de 2 minutos, considerando-se como nível superior aquele que se repetiu em três estimulações sucessivas.

A avaliação da intensidade do bloqueio motor foi realizada através do método proposto por Bromage ${ }^{13}$.

Na fase de recuperação pós-anestésica, foram pesquisados e anotados em protocolo:

a) Tempo da primeira queixa espontânea de dor - decorrido entre a injeção do AL e o momento em que a paciente apresentou a primeira queixa espontânea de dor no lo- 
cal operado, necessitando de analgésico por via sistêmica;

b) Tempo de regressão completa do bloqueio motor - decorrido entre a injeção do AL e bloqueio motor grau zero na escala de Bromage.

Foi pesquisada e anotada em protocolo a ocorrência dos seguintes eventos adversos: PAS $<65 \mathrm{mmHg}$ e/ou PAM $<50$ $\mathrm{mmHg}$; bradicardia; dessaturação do sangue arterial; dor à injeção do AL; tremores; náuseas e vômitos; pruridos. Sempre que ocorreu redução da PAS a níveis inferiores a 65 $\mathrm{mmHg}$ e/ou da PAM a níveis inferiores a $50 \mathrm{mmHg}$, administrou-se vasopressor (etilfedrina) por via venosa. Foi considerada como bradicardia a redução da FC a valor igual ou inferior a $50 \mathrm{bpm}$, administrando-se nesta eventualidade atropina, por via venosa.

Considerou-se como dessaturação do sangue arterial a ocorrência de $\mathrm{SpO}_{2} \leq 85 \%$ por 30 seg ou mais. Os tremores, quando ocorreram, foram tratados com meperidina 20 a 30 mg por via venosa; náuseas e vômitos, com ondansetron 8 mg pela mesma via. Observada a primeira queixa espontânea de dor no pós-operatório, administrou-se diclofenaco 75 mg por via muscular.

A comparação das médias obtidas para os parâmetros hemodinâmicos foi feita através de análise de variância para medidas em série (ANOVA), com nível de significância $p<$ 0,005 .

\section{RESULTADOS}

Os dados demográficos das pacientes estão expressos na tabela I. Onze pacientes (18,3\%) faziam uso crônico de um ou mais destes medicamentos: levotiroxina, paroxetina, hidroclorotiazida, furosemida, bromazepam, enalapril, cloroquina, omeprazol, lamivudina, nevirapina.

Tabela I - Dados Demográficos

\begin{tabular}{lc}
\hline Idade $($ anos) & $38,0 \pm 11,6$ \\
& $(18-62)$ \\
Peso $(\mathrm{kg})^{*}$ & $60,1 \pm 9,1$ \\
& $(46-96)$ \\
Estado físico $\left(\mathrm{n}^{\circ} \mathrm{e} \%\right)$ & \\
ASA I & $50(83,3 \%)$ \\
ASA II & $10(16,7 \%)$ \\
\hline
\end{tabular}

* Valores expressos em Média \pm DP e Amplitude

Adistribuição das pacientes por tipos de cirurgias realizadas está expressa na tabela II. Os procedimentos de lipoaspiração foram realizados sobre diversas regiões corporais combinadas: tórax, abdômen, quadris, coxas. As cirurgias foram realizadas com as pacientes em posição horizontal, eventualmente com elevação do dorso de no máximo $30^{\circ}$.
Tabela II - Distribuição das Pacientes por Tipos de Cirurgias

\begin{tabular}{lc}
\hline Cirurgias & $N^{0}$ de Casos $(\%)$ \\
\hline LA & $19(31,7)$ \\
LA + AP & $3(5,0)$ \\
LA + PG & $11(18,3)$ \\
LA + MP & $10(16,7)$ \\
LA + MP + AP & $12(20,0)$ \\
MP + AP & $5(8,3)$ \\
Total & $60(100,0)$ \\
\hline
\end{tabular}

LA = Lipoaspiração; $\mathrm{AP}=$ Abdominoplastia; $\mathrm{PG}=$ Prótese Glútea; $\mathrm{MP}=$ Mamaplastia

A média de duração dos procedimentos foi $277,9 \pm 55,3 \mathrm{~min}$ (extremos de 150 e $390 \mathrm{~min}$ ).

O nível de punção do espaço peridural foi $\mathrm{T}_{9}-\mathrm{T}_{10}$ em 50 pacientes (83,3\%) e $\mathrm{T}_{10}-\mathrm{T}_{11}$ em 10 (16,7\%). Foi necessária a administração de dose adicional do $\mathrm{AL}$ em 17 pacientes $(28,3 \%)$.

A média obtida para o tempo de latência foi 9,1 $\pm 8,2 \mathrm{~min}$ (extremos de 6 e $12 \mathrm{~min}$ ). O nível superior de bloqueio sensorial foi $\mathrm{T}_{2}$ em 52 pacientes (86,6\%), $\mathrm{C}_{4}$ em $4(6,6 \%)$ e $\mathrm{T}_{3}$ em 4 $(6,6 \%)$. Na direção caudal, o bloqueio sensorial atingiu os dermátomos sacros, permitindo a realização de procedimentos na região glútea.

Na figura 1 estão representados os graus de bloqueio motor obtidos, com os respectivos percentuais de pacientes.

A média obtida para o tempo da primeira queixa espontânea de dor foi $965,1 \pm 371,3 \mathrm{~min}$ (extremos de 510 e $1440 \mathrm{~min}$ ). A média para o tempo de regressão completa do bloqueio motor foi $377,9 \pm 68,5 \mathrm{~min}$ (extremos de 300 e $600 \mathrm{~min}$ ).

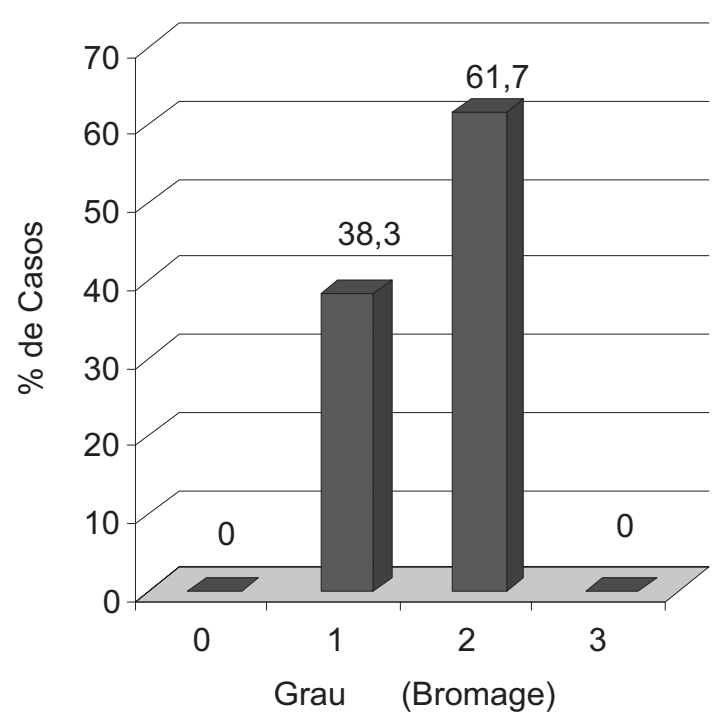

Figura 1 - Intensidade do Bloqueio Motor

Revista Brasileira de Anestesiologia Vol. 52, № 2, Março - Abril, 2002 
A evolução dos valores médios de PAS, PAD e PAM em sete momentos da observação está representada na figura 2, e a da FC na figura 3. Em ambos os casos, as médias decresceram significativamente em relação ao controle a partir dos 15 minutos.

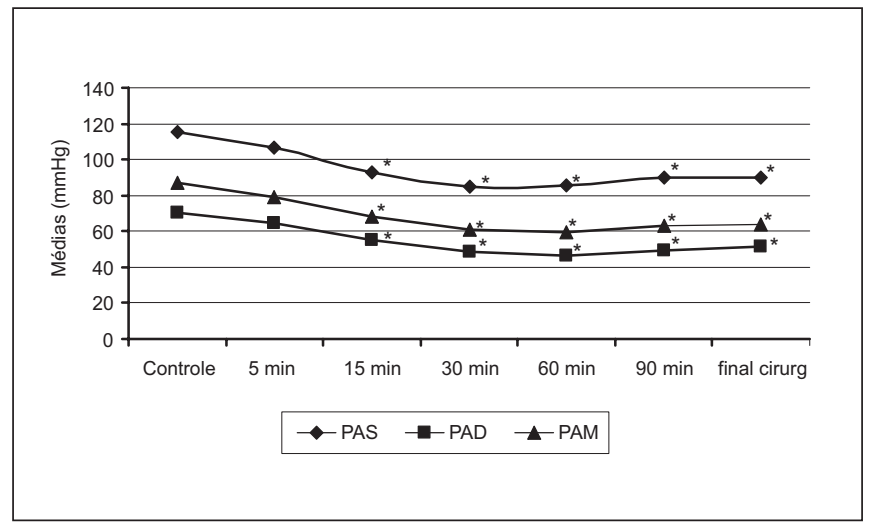

Figura 2 - Evolução dos Valores Médios da PAS, PAD e PAM * Diferença significativa em relação ao controle

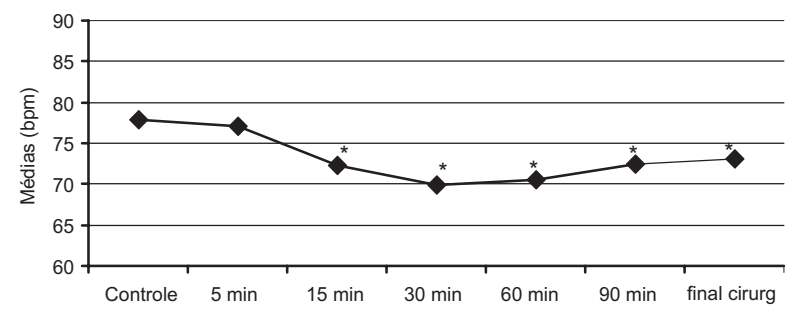

Figura 3 - Evolução dos Valores Médios da FC

* Diferença significativa em relação ao controle

Na tabela lll está expressa a incidência de eventos adversos. Não foi necessária a administração de sangue ou derivados em nenhuma paciente, no per ou no pós-operatório.

Tabela III - Incidência de Eventos Adversos

\begin{tabular}{lcc}
\hline Eventos & $\mathrm{N}^{\circ}$ de Casos & $\%$ \\
\hline PAS < 65 e/ou PAM < 50 mmHg & 13 & 21,7 \\
Bradicardia & 4 & 6,7 \\
Dessaturação do sangue arterial & 1 & 1,7 \\
Dor à injeção do AL & 1 & 1,7 \\
Tremores & 8 & 13,3 \\
Náuseas e vômitos & 1 & 1,7 \\
Pruridos & 0 & 0 \\
\hline
\end{tabular}

Revista Brasileira de Anestesiologia

Vol. 52, № 2, Março - Abril, 2002
A qualidade do bloqueio obtido foi adequada para a realização das cirurgias programadas em todas as pacientes, não sendo necessária complementação com outro método de analgesia.

\section{DISCUSSÃo}

Os resultados mostraram que o bloqueio peridural torácico e lombar extenso com ropivacaína a $0,5 \%$, associado à sedação com propofol em infusão venosa contínua, proporciona anestesia de boa qualidade e segura para cirurgias plásticas combinadas envolvendo diversas regiões corporais.

A adição de um opióide (no caso, o sufentanil) ao AL parece desempenhar papel importante na obtenção não só de qualidade como de algum grau de analgesia pós-operatória, fato já apontado por outros autores ${ }^{14}$. Além disso o opióide em pequenas doses depositado no espaço peridural torácico parece contribuir para a sedação ${ }^{15,16}$, diminuindo assim as necessidades de propofol para obtê-la.

O bloqueio peridural torácico possui efeitos bem documentados sobre o sistema cardiovascular, que resultam basicamente do bloqueio seletivo da inervação simpática cardíaca $\left(T_{1}-T_{5}\right)^{17}$. Estes efeitos são benéficos no sentido de diminuir a excitação cardiovascular, reduzindo o consumo de oxigênio pelo miocárdio em função do controle de pressão arterial sistólica e da freqüência cardíaca. As implicações clínicas são múltiplas, compreendendo a redução das respostas excitatórias cardiovasculares à intubação traqueal ${ }^{18}$, o controle da isquemia miocárdica perioperatória em coronariopatas ${ }^{19}$ e, no nosso caso, a obtenção de menor sangramento em cirurgia plástica com anestesia hipotensiva.

A taquicardia é um dos fatores que mais contribuem para o aumento do débito cardíaco e o sangramento durante a cirurgia. O controle deste fator mostrou-se adequado com a técnica, conforme se pode observar na figura 3 . Convém assinalar que a absorção sistêmica do AL após administração por via peridural pode resultar em depressão direta do miocárdio e bradicardia, configurando o potencial cardiotóxico desta classe de drogas. Isto é particularmente importante no caso da bupivacaína e parece ser responsável por casos de falência circulatória relatados com este $A L$, mormente na área obstétrica ${ }^{20,21}$. A ropivacaína possui potencial cardiotóxico significativamente menor que o da bupivacaína ${ }^{22,23}$, o que diminui a possibilidade de falência circulatória pela somação de efeitos com o bloqueio simpático pela peridural. De qualquer maneira, ocorreram em quatro pacientes $(6,6 \%$ dos casos) episódios de bradicardia com redução da FC a valores inferiores a $50 \mathrm{bpm}$, prontamente revertidos pela administração de atropina.

Nos casos de lipoaspiração, é praticada pelo cirurgião a infiltração das áreas a serem aspiradas, com grandes volumes de solução fisiológica contendo lidocaína e adrenalina extremamente diluídas (solução tumescente). A baixa concentração e a perfusão tecidual diminuída alteram a farmacologia clínica da lidocaína na solução tumescente, elevando a dose máxima do AL em até 7 a 8 vezes a recomendada em infiltra- 
ção convencional ${ }^{24}$. Com efeito, nos casos estudados não ocorreu nenhuma manifestação de toxicidade por absorção sistêmica do AL. Apesar da concentração extremamente baixa, é possível que a lidocaína contribua com algum grau de analgesia nas áreas infiltradas.

Após a instalação do bloqueio, houve diminuição da pressão arterial conforme se pode observar na figura 2. É importante salientar que este efeito foi desejado, configurando técnica de anestesia hipotensiva com a finalidade de diminuir o sangramento operatório. APAS foi mantida em valores não inferiores a $65 \mathrm{mmHg}$ e a PAM em valores não inferiores a 50 $\mathrm{mmHg}$, considerados seguros do ponto de vista da perfusão cerebral e miocárdica ${ }^{1,2}$. Ocorreram em treze pacientes ( $21,7 \%$ dos casos) episódios de diminuição da PAS e da PAM a valores abaixo dos desejados, os quais foram fácil e prontamente abolidos pela administração de etilfedrina. Este é um vasopressor de ação mista do grupo da efedrina ${ }^{25}$ que, como esta, é bem indicado na reversão da hipotensão causada por bloqueio simpático em anestesia regional, com a vantagem de não provocar o mesmo grau de elevação da FC que acompanha o uso da efedrina.

A incidência de hipotensão arterial e de bradicardia tratadas farmacologicamente neste estudo foi inferior à encontrada por outros autores que utilizaram lidocaína ou bupivacaína em bloqueio peridural torácico ${ }^{26,27}$

É importante lembrar que, mesmo na vigência de bloqueio peridural alto, existem mecanismos de compensação que garantem alguma atividade simpática. Com efeito, estudos mostraram que a anestesia peridural com nível superior de bloqueio em $\mathrm{C}_{8}$ não se acompanha de redução significativa dos níveis plasmáticos de adrenalina ${ }^{28}$; a manutenção destes níveis deve compensar pelo menos parcialmente os efeitos depressores circulatórios da anestesia peridural.

Aassociação do bloqueio peridural com sedação pelo propofol em infusão contínua nas doses utilizadas proporcionou maior conforto para a paciente e não resultou em ulterior deterioração dos parâmetros circulatórios, o que está de acordo com observações anteriores ${ }^{29,31}$.

Do mesmo modo, não se observou dessaturação do sangue arterial a níveis de $\mathrm{SpO}_{2} \leq 85 \%$ no transcorrer do procedimento, exceto em uma paciente. Sabe-se que o bloqueio peridural torácico com soluções concentradas de bupivacaína provoca redução moderada da capacidade vital e do fluxo inspiratório máximo, devido provavelmente a um certo grau de relaxamento dos músculos intercostais ${ }^{32}$. Entretanto, a utilização de soluções menos concentradas de um AL como a ropivacaína, que produz bloqueio motor de menor intensidade quando comparado ao da bupivacaína, pode diminuir a possibilidade de comprometimento significativo da musculatura respiratória. Por outro lado, já foi relatado que a sedação com infusão de propofol na velocidade utilizada no nosso estudo é segura do ponto de vista respiratório ${ }^{31}$.

O tempo decorrido desde a injeção do AL e do sufentanil até que se manifestou a necessidade de analgésico por via sistêmica foi apreciável, podendo ter contribuído para isto a analgesia "preemptiva" proporcionada pelo sufentanil, efeito já demonstrado para outros opióides por via peridural especialmente em cirurgias mamárias e de membros ${ }^{33}$.

Ocorreram tremores pós-operatórios em oito pacientes (13,3\% dos casos), todas submetidas à lipoaspiração. Foram devidos provavelmente à diminuição da temperatura corporal, secundária à perda de calor para o meio ambiente facilitada pela vasodilatação da peridural, e ao resfriamento corporal pela infiltração das áreas aspiradas com solução fisiológica fria. Foram abolidos pela administração de meperidina, uma substância que produz redução significativa do limiar da temperatura corporal capaz de disparar o mecanismo termorregulador ${ }^{34}$.

Concluímos que o bloqueio peridural torácico com solução de ropivacaína a $0,5 \%$ adicionada de sufentanil, associado à sedação com propofol em infusão venosa contínua, constitui método de anestesia hipotensiva seguro e de boa qualidade para cirurgias plásticas combinadas envolvendo mama, abdômen, glúteos e lipoaspiração. Não é um procedimento simples: seu bom êxito depende da habilidade do anestesiologista, da sua capacidade para dominar os efeitos do bloqueio sobre os parâmetros circulatórios e respiratórios, da monitorização contínua destes parâmetros e do entrosamento de toda a equipe cirúrgica. Só a conjugação destes fatores pode garantir os bons resultados e a segurança do paciente.

\section{Thoracic Epidural Anesthesia with Ropivacaine for Plastic Surgery}

José Roberto Nociti, TSA, M.D., Paulo Sérgio Mateus Serzedo, TSA, M.D., Eduardo Barbin Zuccolotto, TSA, M.D., Raul Gonzalez, M.D.

\section{INTRODUCTION}

Plastic surgery is one of the surgical specialties which benefits most from induced hypotension. For a long time it has been observed that maintaining systolic blood pressure not higher than $65 \mathrm{mmHg}$ and mean blood pressure not higher than $50 \mathrm{mmHg}$ is safe and significantly decreases surgical bleeding ${ }^{1,2}$. This observation has evolved to the concept of hypotensive anesthesia ${ }^{3}$ meaning anesthetic techniques aiming at safely decreasing bleeding. One of these techniques is the epidural block, thanks to preload and systemic vascular resistance decrease and, in the case of thoracic epidural block, also a decrease in myocardial contractility secondary to the blockade of sympathetic fibers to the heart ${ }^{4,5}$.

On the other hand, good results have been obtained with ropivacaine, a new local anesthetic (LA) drug of the pipecholxylidide group, in epidural anesthesia ${ }^{6}$, with emphasis to less motor block ${ }^{7}$ and lower cardiovascular and nervous system toxicity, as compared to bupivacaine ${ }^{8}$.

This non-comparative study aimed at evaluating the results of thoracic epidural block with $0.5 \%$ ropivacaine associated to sufentanil and propofol continuous infusion sedation in plastic surgery.

Revista Brasileira de Anestesiologia Vol. 52, № 2, Março - Abril, 2002 


\section{METHODS}

After the Hospital's Ethics Committee approval, participated in this study 60 patients according to the following selection criteria:

1. Female gender, aged 18 years or above;

2. Physical status ASA I or II;

3. Scheduled for combined elective plastic surgeries of the breast, abdomen, gluteus and liposuction.

Patients were premedicated with oral flunitrazepam (30 mg) 60 minutes before surgery.

After a large vein catheterization and starting a $5 \%$ glucose solution infusion, epidural space puncture was performed in $T_{9}-T_{10}$ or $T_{10}-T_{11}$ with patients in the sitting position. Epidural space was identified through the loss of resistance to air technique and $40 \mathrm{ml}$ of $0.5 \%$ ropivacaine (corresponding to 200 $\mathrm{mg}$ of $L A$ ) associated to $15 \mu \mathrm{g}$ sufentanil was administered at the speed of $5 \mathrm{ml} \cdot \mathrm{min}^{-1}$. Next, a catheter was introduced in the epidural space 8 to $10 \mathrm{~cm}$ in the cephalad direction for the administration of additional ropivacaine doses $(15 \mathrm{ml}$ of $0.5 \%$ solution corresponding to $75 \mathrm{mg}$ ), whenever required by surgery duration.

After epidural block, sedation was induced with intravenous midazolam $\left(0.10 \mathrm{mg} \cdot \mathrm{kg}^{-1}\right)$ followed by propofol continuous infusion at the speed of 40 to $50 \mu \mathrm{g} \cdot \mathrm{kg}^{-1} \cdot \mathrm{min}^{-1}$ throughout the procedure. An oxygen flow of $1-2$ L. min $^{-1}$ was routinely administered through a catheter introduced in an oropharyngeal cannula (Guedel).

The following parameters were non-invasively monitored: systolic blood pressure (SBP), diastolic blood pressure (DBP), mean blood pressure (MBP), heart rate (HR), ECG, respiratory rate $(\mathrm{RR})$ and pulse oximetry $\left(\mathrm{SpO}_{2}\right)$. Values were recorded in the following moments:

- Before epidural block (control);

- 5 minutes after LA injection;

- 15 minutes after injection;

- 30 minutes after injection;

- At every 30 minutes from then on.

Intraoperative fluids were administered according to the rule proposed by Jenkins and Giesecke ${ }^{9}$, for larger superficial surgeries: 8 to $10 \mathrm{ml} \cdot \mathrm{kg}^{-1} \cdot \mathrm{h}^{-1}$, starting with $5 \%$ glucose solution until the volume of $500 \mathrm{ml}$ and continuing with lactated Ringer's solution and $5 \%$ glucose, never exceeding the total dose of $125 \mathrm{~g}$ glucose. For wet liposuction, the surgeon has infiltrated the areas to be sucked with saline containing $0.1 \%$ lidocaine and epinephrine $1: 1.000 .000^{10}$. Since more than $80 \%$ of the infiltrated volume ends up absorbed by circulation 11,12 , this volume was subtracted from the volume calculated by Jenkins and Giesecke's rule and lower than expected final fluid volume was administered.

Sensory block was evaluated as follows:

Revista Brasileira de Anestesiologia

Vol. 52, No 2, Março - Abril, 2002 a) Onset time - time elapsed between end of injection and absence of pain at stimulation with a surgical clamp in the sensory area corresponding to the puncture level;

b) Upper block - by skin stimulation with a surgical clamp at 2-minute intervals, considering the upper level the one abtained repeatedly in 3 consecutive stimulations.

Motor block intensity was evaluated by Bromage's method ${ }^{13}$ During post-anesthetic recovery, the following parameters were studied and recorded:

a) Time for first spontaneous pain complaint - elapsed between LA injection and the moment patients referred their first spontaneous pain complaint at the operated site, needing systemic analgesia;

b) Time for total motor block regression - elapsed between LA injection and motor block zero according to Bromage's scale.

The incidence of the following adverse effects was observed and recorded: SBP < $65 \mathrm{mmHg}$ and/or $\mathrm{MBP}<50 \mathrm{mmHg}$; bradycardia; arterial blood desaturation; pain at LAinjection; shivering; nausea and vomiting; pruritus. Whenever SBP fell below $65 \mathrm{mmHg}$ and/or MBP fell below $50 \mathrm{mmHg}$, an intravenous vasopressor (ethylphedrine) was administered. Bradycardia was considered as HR decrease to $50 \mathrm{bpm}$ or below and was treated with intravenous atropine.

Arterial blood desaturation was defined as $\mathrm{SpO}_{2} \leq 85 \%$ for 30 seconds or more. Shivering was treated with 20 to $30 \mathrm{mg}$ intravenous meperidine; nausea and vomiting with $8 \mathrm{mg}$ intravenous ondansetron. First spontaneous pain complaint after surgery was treated with 75 mg muscular dichlophenac. Mean hemodynamic parameters were compared by analysis of variance for serial measurements (ANOVA), considering significant a $p<0.005$.

\section{RESULTS}

Demographics are shown in table I. Eleven patients (18.3\%) were in chronic use of one or more of the following drugs: levotiroxine, paroxetine, hydrochlorothiazide, furosemide, bromazepam, enalapril, chloroquine, omeprazol, lamivudine, nevirapine.

Table I - Demographics Data

\begin{tabular}{lc}
\hline Age $(\text { years })^{*}$ & $38.0 \pm 11.6$ \\
& $(18-62)$ \\
Weight $(\mathrm{kg})^{*}$ & $60.1 \pm 9.1$ \\
& $(46-96)$ \\
Physical status $\left(\mathrm{n}^{\circ}\right.$ and \%) & \\
ASA I & $50(83.3 \%)$ \\
ASA II & $10(16.7 \%)$ \\
\hline * Values expressed in Mean \pm SD and Amplitude
\end{tabular}


Patients distribution per surgery type is shown in table II. Liposuction was performed in several combined body regions: chest, abdomen, hips and thighs. Surgeries were performed with patients in the horizontal position with maximum dorsal elevation of $30^{\circ}$.

Mean procedure duration was $277.9 \pm 55.3 \mathrm{~min}$ (extremes of 150 and $390 \mathrm{~min}$ ). Epidural space puncture level was $\mathrm{T}_{9}-\mathrm{T}_{10}$ in 50 patients $(83.3 \%)$ and $\mathrm{T}_{10}-\mathrm{T}_{11}$ in 17 patients $(28.3 \%)$.

Table II - Patients Distribution per Surgery Type

\begin{tabular}{lc}
\hline Surgeries & $N^{\circ}$ of Cases $(\%)$ \\
\hline LS & $19(31.7)$ \\
LS + AP & $3(5.0)$ \\
LS + GP & $11(18.3)$ \\
LS + MP & $10(16.7)$ \\
LS + MP + AP & $12(20.0)$ \\
MP + AP & $5(8.3)$ \\
Total & $60(100.0)$ \\
\hline
\end{tabular}

LS = Liposuction; AP = Abdominoplasty; GP = Gluteal Prosthesis; MP = Mammaplasty

Mean onset time was $9.1 \pm 8.2 \mathrm{~min}$ (extremes of 6 and $12 \mathrm{~min}$ ). Upper sensory block level was $\mathrm{T}_{2}$ in 52 patients (86.6\%), $\mathrm{C}_{4}$ in $4(6.6 \%)$ and $\mathrm{T}_{3}$ in $4(6.6 \%)$. Caudal sensory block has reached sacral dermatomes allowing for procedures in the gluteal region.

Motor block levels and patient percentages are shown in figure 1.

Mean time for first spontaneous pain complaint was $965.1 \pm$ $371.3 \mathrm{~min}$ (extremes of 510 and $1440 \mathrm{~min}$ ). Mean total motor block regression time was $377.9 \pm 68.5 \mathrm{~min}$ (extremes of 300 and $600 \mathrm{~min}$ ).

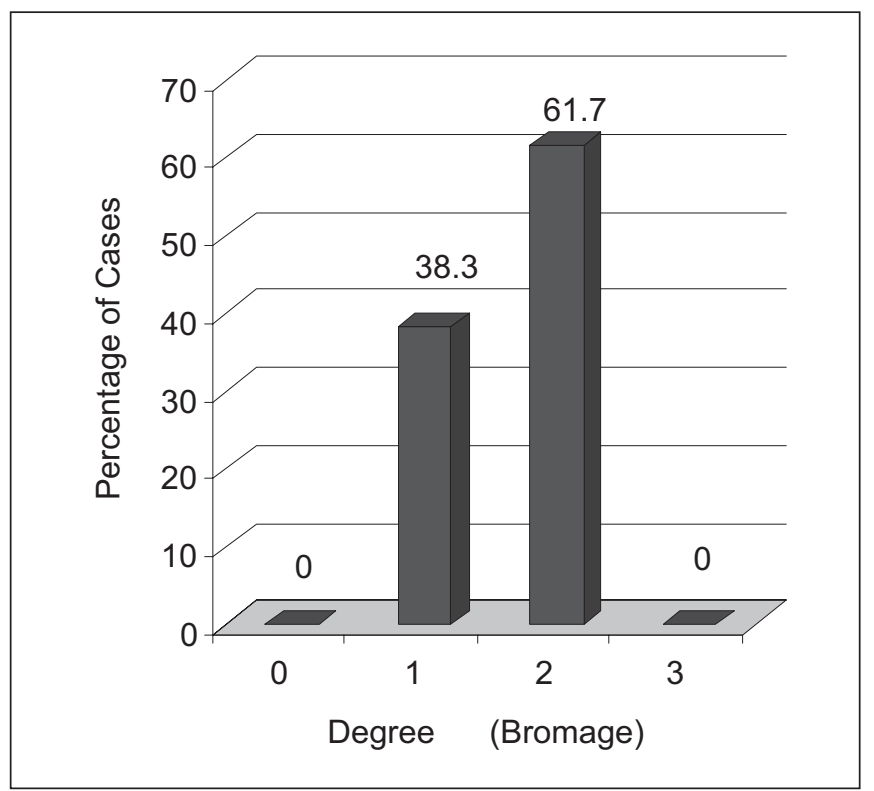

Figure 1 - Motor Block Intensity
SBP, DBP and MBP mean values in seven observation moments are shown in figure 2 and mean $\mathrm{HR}$ is shown in figure 3 . Means have significantly decreased after 15 minutes for both groups as compared to control.

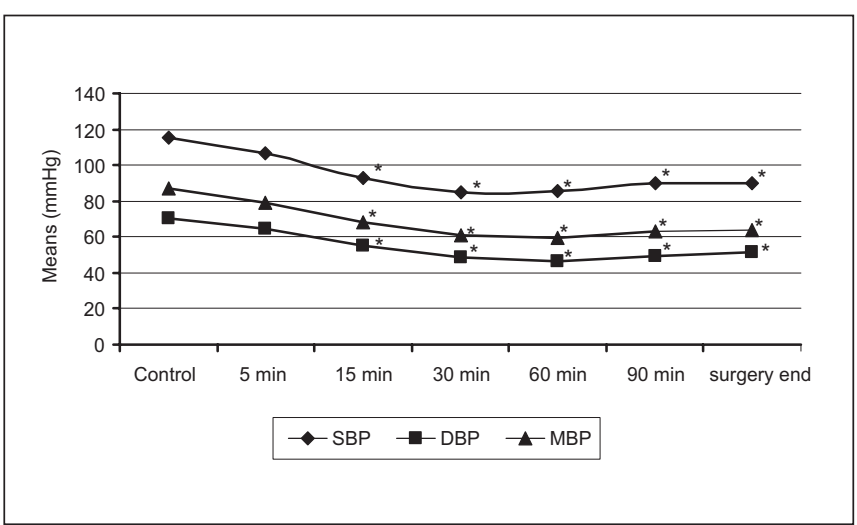

Figure 2 - SBP, DBP and MBP Mean Values Evolution * Significant difference as compared to control

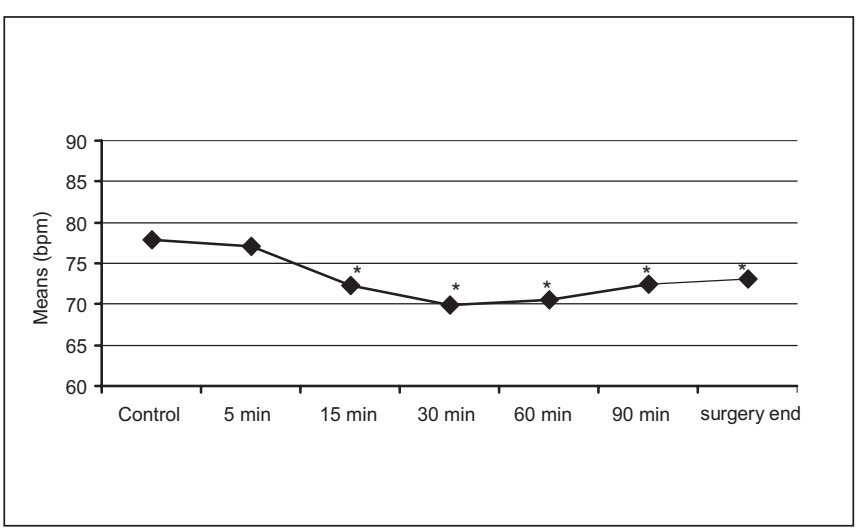

Figure 3 - HR Mean Values Evolution

* Significant difference as compared to control.

Adverse events are shown in table III. There has been no need for perioperative blood or by-products administration. Blockade was adequate for all patients with no need for additional analgesia.

Table III - Incidence of Adverse Events

\begin{tabular}{lcc}
\hline Events & $\mathrm{N}^{\circ}$ of cases & $\%$ \\
\hline $\mathrm{SBP}<65$ and/or $\mathrm{MBP}<50 \mathrm{mmHg}$ & 13 & 21.7 \\
Bradycardia & 4 & 6.7 \\
Arterial blood desaturation & 1 & 1.7 \\
Pain at LA injection & 1 & 1.7 \\
Shivering & 8 & 13.3 \\
Náusea and vomiting & 1 & 1.7 \\
Pruritus & 0 & 0 \\
\hline
\end{tabular}




\section{DISCUSSION}

Results have shown that extensive epidural thoracic and lumbar blockade with $0.5 \%$ ropivacaine associated to propofol continuous infusion sedation is a good and safe anesthetic technique for combined plastic surgeries involving different body regions.

The addition of an opioid (sufentanil in this case) to LA seems to play an important role in the quality of the blockade and in postoperative analgesia, which is in line with other authors ${ }^{14}$. Moreover, small opioid doses in the thoracic epidural space seem to contribute to sedation ${ }^{15,16}$, thus decreasing the need for propofol.

Thoracic epidural block has well known effects on the cardiovascular system, resulting in a selective sympathetic heart enervation blockade $\left(T_{1}-T_{5}\right)^{17}$. These are beneficial effects because they decrease cardiovascular excitation and reduce myocardial oxygen consumption by controlling systolic blood pressure and heart rate. There are multiple clinical implications, such as decrease in cardiovascular excitatory responses to tracheal intubation ${ }^{18}$, perioperative myocardial ischemia control in cardiac diseases ${ }^{19}$ and, in our case, less bleeding in plastic surgeries with hypotensive anesthesia.

Tachycardia is a major factor for increasing cardiac output and bleeding during surgery. The technique has adequately controlled such factor, as shown in figure 3 . It is worth mentioning that systemic LAabsorption after epidural administration may result in direct myocardial depression and bradycardia, configuring the cardiotoxic potential of such drugs. This is especially important with bupivacaine and seems to be responsible for circulatory failures reported with this LA, especially in obstetrics ${ }^{20,21}$. Ropivacaine is significantly less cardiotoxic than bupivacaine ${ }^{22,23}$, thus decreasing the chances for circulatory failure caused by the addition of effects with sympathetic block. However, there were 4 bradycardia episodes (6.6\% of cases) with HR decreases to $50 \mathrm{bpm}$ or less, promptly reverted with atropine.

Areas to be sucked during liposuction are infiltrated by the surgeon with large volumes of saline with highly diluted lidocaine and epinephrine (tumescent solution). Low concentration and decreased tissue perfusion change lidocaine's clinical pharmacology in the tumescent solution, increasing the highest LA dose in up to 7 to 8 times the recommended dose for traditional infiltration ${ }^{24}$. Indeed, there has been no toxicity by systemic LAabsorption in our study. Regardless of the extremely low concentration, lidocaine may contribute to a certain degree of analgesia in the infiltrated areas.

After blockade, there has been a decrease in blood pressure, as shown in figure 2. It is important to highlight that this was a desired effect of hypotensive anesthesia aiming at decreasing surgical bleeding. SBP was maintained in values not below $65 \mathrm{mmHg}$ and MBP in values not below $50 \mathrm{mmHg}$, which are considered safe from brain and myocardial perfusion viewpoint $^{1,2}$.SBP and MBP decreases below desirable values were observed in 13 patients $(21.7 \%)$ and were easily and promptly treated with ethylphedrine. This is a mixed action vasopressor of the ephedrine group ${ }^{25}$, constituting a good choice to revert hypotension caused by sympathetic blockade during regional anesthesia, with the additional advantage of not causing the same HR increase following the administration of ephedrine.

The incidence of pharmacologically treated hypotension and bradycardia in our study was lower than what has been reported by other authors using lidocaine or bupivacaine in thoracic epidural blocks ${ }^{26,27}$.

It is important to remind that, even during high epidural blocks, there are compensating mechanisms which allow for some sympathetic activity. Indeed, studies have shown that epidural anesthesia with upper blockade level in $\mathrm{C}_{8}$ is not followed by significant epinephrine plasma level decrease ${ }^{28}$; the maintenance of such levels should compensate, at least partially, circulatory depressing effects of epidural anesthesia.

The association of epidural block with propofol continuous infusion sedation in the doses used in this study has provided more comfort to patients and did not result in circulatory parameters deterioration, which is in line with previous observations ${ }^{29,31}$.

Similarly, there has been no arterial blood oxygen desaturation to $\mathrm{SpO}_{2} \leq 85 \%$ throughout the procedure, except for one patient. It is known that thoracic epidural block with concentrated bupivacaine solutions causes a moderate decrease in vital capacity and maximum inspiratory flow, probably due to a certain degree of intercostal muscles relaxation ${ }^{32}$. However, less concentrated solutions of an LA such as ropivacaine, which induces a less intense motor block as compared to bupivacaine, may decrease the chances of significant involvement of respiratory muscles. On the other hand, it has already been reported that sedation with propofol infusion at the speed used in our study is safe for the respiratory system ${ }^{31}$.

Time elapsed between LA and sufentanil injection to the first systemic analgesic need was considerable and may have been influenced by the preemptive analgesia provided by sufentanil; this effect has already been observed for other epidural opioids, especially for breast and limbs surgery ${ }^{33}$.

Postoperative shivering was seen in 8 patients (13.3\%), all submitted to liposuction, and was probably caused by the decrease in body temperature, secondary to heat loss to the environment facilitated by epidural vasodilation, and to body cooling by infiltrating sucked areas with cold saline. Shiverings was treated with meperidine, which produces significant decrease in body temperature threshold and is able to trigger the thermoregulating mechanism ${ }^{34}$.

The conclusion was that thoracic epidural block with $0.5 \%$ ropivacaine plus sufentanil, associated to propofol continuous infusion sedation is a safe and good hypotensive anesthesia method for combined plastic surgeries involving breast, abdomen, gluteus and liposuction. It is not a simple procedure; its success depends on anesthesiologists' skills, on their ability to deal with blockade effects on circulatory and 
respiratory parameters, on the constant monitoring of such parameters and on the synchronism of the surgical team. The concomitance of all those factors is paramount for assuring good results and patient safety.

\section{REFERÊNCIAS - REFERENCES}

01. Lawson NW, Thompson DS, Nelson CL et al - A dosage nomogram for sodium nitroprusside-induced hypotension under anesthesia. Anesth Analg, 1976;55:574-580.

02. Thompson GE, Miller RD, Stevens WC - Hypotensive anesthesia for total hip arthroplasty: a study of blood loss and organ function (brain, heart, liver, and kidney). Anesthesiology, 1978;48:91-96

03. Nocite JR - Hipotensão induzida ou anestesia hipotensiva? Rev Bras Anestesiol, 1988;38:391-392.

04. Adams AP - Techniques of vascular control for deliberate hypotension during anaesthesia. $\mathrm{Br} \mathrm{J}$ Anaesth, 1975;47: 777-784.

05. Vieira JL, Vanetti LFA - Hipotensão arterial induzida durante cirurgia: fisiologia, técnica, riscos. Rev Bras Anestesiol, 1982;32:185-206.

06. Nociti JR, Cagnolati CA, Nunes AMM et al - Ropivacaína a $0,75 \%$ e $1 \%$ em anestesia peridural para cirurgia: estudo comparativo. Rev Bras Anestesiol, 1998;48:169-176.

07. Zaric D, Nydahl P, Philipson L et al - The effect of continuous lumbar epidural infusion of ropivacaine $(0.1 \%, 0.2 \%$ and $0.3 \%)$ and $0.25 \%$ bupivacaine on sensory and motor blockade in volunteers : a double-blind study. Reg Anesth, 1996;21:14-22.

08. Knudsen K, Suurkula MB, Blomberg S et al - Central nervous and cardiovascular effects of i.v. infusions of ropivacaine, bupivacaine and placebo in volunteers. $\mathrm{Br} \mathrm{J}$ Anaesth, 1997;78:507-514.

09. Jenkins MT, Giesecke AH - Balanced Salt Solution in Clinical Anesthesia. Refresher Courses in Anesthesiology, Philadelphia, The ASA Inc, 1974;2:107-116.

10. Sommer B, Breuninger $\mathrm{H}$ - Composition of the Solution for Tumescent Anesthesia, em: Hanke CW, Sommer B, Sattler G - Tumescent Local Anesthesia. Berlin Heidelberg, Springer-Verlag, 2001;9-13.

11. Klein JA - The tumescent technique for liposuction surgery. The Amer J Cosmet Surg, 1987;4:263-267.

12. Klein JA - Tumescent technique for local anesthesia improves safety in large-volume liposuction. Plast Reconstr Surg, 1993;92:1085-1098.

13. Bromage PR - Epidural Analgesia, $1^{\text {st }}$ Ed, Philadelphia, WB Saunders, 1978;144.

14. Carli F, Klubien K- Thoracic epidurals: is analgesia all we want? Can J Anaesth, 1999;46:409-414

15. Bromage PR, Camporesi EM, Leslie J - Epidural narcotics in volunteers: sensitivity to pain and to carbon dioxide. Pain, 1980;9:145-160.

16. Bromage PR - Thoracic epidurals: reply to Hough (Letter). Reg Anesth Pain Med, 1999;24:273.

17. Meissner A, Rolf $\mathrm{N}$, Van Aken $\mathrm{H}$ - Thoracic epidural anesthesia and the patient with heart disease : benefits, risks, and controversies. Anesth Analg, 1997;85:517-528.

18. Watwil M, Sundberg A, Olsson J et al - Thoracolumbar epidural anaesthesia blocks the circulatory response to laryngoscopy and intubation. Acta Anaesthesiol Scand, 1987;31:529-531.
19. Koch M, Blomberg S, Emanuelsson H - Thoracic epidural anesthesia improves global and regional left ventricular function during stress-induced myocardial ischemia in patients with coronary artery diseases. Anesth Analg, 1990;7:625-630.

20. Reiz S, Nath S - Cardiotoxicity of local anaesthetic agents. Br J Anaesth, 1986;58:736-746.

21. Albright CA - Cardiac arrest following regional anesthesia with etidocaine or bupivacaine. Anesthesiology, 1979;51:285-287.

22. Reiz S, Haggmark S, Johansson $G$ et al - Cardiotoxicity of ropivacaine - a new amide local anaesthetic agent. Acta Anaesthesiol Scand, 1989;33:93-98.

23. Pitkanen M, CovinoBG, Feldman HS et al - Chronotropic and inotropic effects of ropivacaine, bupivacaine, and lidocaine in the spontaneously beating and electrically paced isolated, perfused rabbit heart. Reg Anesth, 1992;17:183-192.

24. Schwarz S, Bergfeld D, Sommer B - Pharmacology, em: Hanke CW, Sommer B, Sattler G - Tumescent Local Anesthesia. Berlin Heidelberg, Springer-Verlag, 2001;14-27.

25. Smith NT, Corbascio AN - The use and misuse of pressor agents. Anesthesiology, 1970;33:58-63.

26. Leão DG - Peridural torácica: estudo retrospectivo de 1240 casos. Rev Bras Anestesiol, 1997;47:138-147.

27. Gouveia MA, Ribeiro RC - Anestesia peridural cervico-torácica. Apreciação clínica. Rev Bras Anestesiol, 1974;24:238-248.

28. Stevens RA, Artusio JD, Kao TC et al - Changes in human plasma catecholamine concentrations during epidural anesthesia depends on the level of block. Anesthesiology, 1991;74: 1029-1034.

29. Herrero E, Del Valle SG, La Quintana B et al - Sedación con propofol durante anestesia locorregional. Rev Esp Anest Rean, 1993;40 (Supl 1):31.

30. Wilson E, Mackenzie N, Grant IS - A comparison of propofol and midazolam by infusion to provide sedation in patients who receive spinal anaesthesia. Anaesthesia, 1988;43:(Suppl):91-94.

31. Nocite JR, Cagnolati CA, Nunes AMM et al - Sedação com propofol durante anestesia peridural. Rev Bras Anestesiol, 1996;46:259-266.

32. Sundberg A, Wattwil M, Arvill A et al - Respiratory effects of high thoracic epidural anaesthesia. Acta Anaesthesiol Scand, 1986;30:215-217.

33. Aida S, Baba H, Yamamura T et al - The effectiveness of preemptive analgesia varies according to the type of surgery : a randomized, double-blind study. Anesth Analg, 1999;89: 711-716.

34. Kurz A, Ikeda T, Sessler DI et al - Meperidine decreases the shivering threshold twice as much as the vasoconstriction threshold. Anesthesiology, 1997;86:1046-1054.

\section{RESUMEN}

Nociti JR, Serzedo PSM, Zuccolotto EB, Gonzalez R - Ropivacaína en Bloqueo Peridural Torácico para Cirugía Plástica

Justificativa y Objetivos - El bloqueo peridural torácico constituye técnica de anestesia hipotensiva, capaz de reducir el sangramiento en el campo operatorio. El presente estudio no-comparativo tiene por finalidad observar los resultados del bloqueo peridural torácico con ropivacaína a 0,5\% asociado a sedación con infusión continua de propofol en cirugía plástica.

Método - Participaron del estudio sesenta pacientes del sexo femenino con edades entre 18 y 62 años, estado físico ASA I ó II, sometidas a cirugías plásticas combinadas envolviendo mama, abdomen, glúteos, lipoaspiración. Después de punción peridural en $T_{9}-T_{10}$ ó $T_{10}-T_{11}$, recibieron $40 \mathrm{ml}$ de solución de ropivacaína a $0,5 \%$ y sufentanil $15 \mu \mathrm{g}$. Dosis subsecuentes de anestésico local fueron administradas a través de catéter 
cuando necesarias. La sedación fue obtenida con infusión venosa continua de propofol 40 a $50 \mu \mathrm{g} \cdot \mathrm{kg}^{-1} \cdot \mathrm{min}^{-1}$. Fueron evaluadas las características de instalación y regresión del bloqueo, la evolución de los parámetros hemodinámicos y respiratorios, la incidencia de eventos adversos.

Resultados - El nivel superior de bloqueo sensorial fue $T_{2}$ en 52 pacientes (86,6\%), $C_{4}$ en $4(6,6 \%)$ e $T_{3}$ en $4(6,6 \%)$. La media para el tiempo de latencia fue 9,1 $\pm 8,2$ minutos. Se obtuvo bloqueo motor grado 2 en $61,7 \%$ de las pacientes y grado 1 en $38,3 \%$. La media para el tiempo de regresión completa del bloqueo motor fue $377,9 \pm 68,5$ minutos. La media para el tiempo de la primera queja espontanea de dolor fue 965,1 \pm 371,3 minutos. Los valores medios de PAS, PAD, PAM y FC decrecieron significativamente en relación al control a partir de los 15 min después de la inyección de anestésico local, caracterizando anestesia hipotensiva. Trece pacientes $(21,7 \%)$ que presentaron PAS $<65 \mathrm{mmHg}$ y/o PAM $<50 \mathrm{mmHg}$ recibieron vasopresor (etilfedrina) y $4(6,7 \%)$ que presentaron $F C<50$ bpm recibieron atropina. No fue necesaria transfusión sanguínea en ningún paciente.

Conclusiones - El bloqueo peridural torácico con ropivacaína a $0,5 \%$ y sufentanil $15 \mu \mathrm{g}$, asociado a la sedación con propofol en infusión continua, constituye método de anestesia hipotensiva de buena calidad para cirugías plásticas combinadas envolviendo mama, abdomen, glúteos y lipoaspiración. La monitorización contínua de los parámetros hemodinámicos y respiratorios y el control de los efectos del bloqueo sobre estos parámetros son indispensables para el suceso y la seguridad de la técnica. 